\title{
Umbilical-Urachus Sinus A variant of Patent Urachus Presentation With Management in Adults- A single Center Experience
}

\author{
Authors \\ Priyabrata Das, Rupali Patnaik, Vaibav Vikas, Shanky Singh \\ Department of Urology, Government Medical College, Trivandrum, India \\ Corresponding Author \\ Shanky Singh \\ RNRA-27, Rajiv Gandhi Nagar, Medical College PO, Trivandrum- 695011
}

\section{Introduction}

The urachus is located pre-peritoneally in the center of a pyramid-shaped space. This space lies with its base on the anterior dome of the bladder and the tip directed toward the umbilicus. The urachal length varies from 3 to $10 \mathrm{~cm}$. It has a diameter of 8 to $10 \mathrm{~mm}$. The urachus can remain either completely open or obliterate partially, leading to the formation of cystic structures at any site throughout its course. The urachal anamoly is usually discovered in childhood, but a late onset in adulthood is always possible. In these cases the clinical presentation is highly variable, and makes diagnosis difficult. This case series presents the various ways of presentation and their management in adults.

\section{Aim and Objective}

To study the mode of presentation and management of urachal sinus in adults.

\section{Material and Methods}

A Case Series

Study Period: 2014 August to 2017 July.

\section{Case Report I}

A $35 \mathrm{yr}$ old male with c/o serous discharge from umbilicus-2 months duration. Initially had pain and swelling around umbilicus with purulent discharge. Was treated with antibiotics elsewhere and referred to us with Ultra sound of abdomen. No history of fever with chills or similar episodes in the past. Patient is a known case of Type I Diabetic on insulin for $10 \mathrm{yrs}$. No history of previous surgeries. Abdominal examination revealed soft non tender, mass of size $2 \times 2 \mathrm{~cm}$ palpable around umbilicus, with mild tenderness Bladder not palpable. Serous discharge from umbilicus with no granulation tissue. The umbilicus looked normal and no peritoneal signs were elicited. Blood biochemistry was normal. Urinalysis was normal and urine culture showed no bacterial growth. Abdominal ultrasonography (US) revealed a $16 \times 15 \mathrm{~mm}$ hypoechoic area beneath the umbilicus in subcutaneous plane connected to umbilicus and tract like structure going down not reaching bladder. CT sinogram reveals a Sinus tract $9.6 \mathrm{~mm}$ long with external opening in umbilicus and connected to above midline swelling. Cystoscopy was performed 
showing no bladder anamolies. The suggested diagnosis was Umbilical-Urachus Sinus. Whole of sinus tract along with abscess cavity and median umbilical ligament with a cuff of dome of bladder removed extraperitonealy preserving the umbilicus. Histopahological report of excised tract also confirmed for urachal tissue. At 18 months postoperatively, Patient was asymptomatic and no abnormalities of the abdominal wall were seen.

\section{Case Report-II}

A 40 yr old man presented to emergency with pain abdomen and fever for 3 days. Pain was moderate to severe in intensity without any radiation. Fever with chills are there for 3 days.

Past history of recurrent umbilical discharge was there which was purulent in nature. Patient was a known case of diabetes on oral hypoglycemic. Abdominal examination revealed swelling around the umbilicus with tenderness, no signs of peritonitis was present. Initial diagnosis was umbilical hernia. Patient refereed to us with Ultrasound of abdomen showing a hypoechoic mass below umbilicus with echogenic particle inside. Blood biochemistry shows leucocytosis $(14,5000)$ with neutrophilia. All other blood parameters are within normal range. Urine culure and sensitivity was sterile. With antibiotic coverage patient was subjected to CECT scan. Which shows 11.3 x $14.5 \times 12.3 \mathrm{~mm}$ well defined midline collection located deep to the umbilicus in preperitoneal space in line with urachus. with few air pockets noted. Urinary bladder normal. Cystoscopy was performed showing no bladder anomalies. Provisional diagnosis was infected urachal sinus. Whole of sinus tract along with abscess cavity and median umbilical ligament with a cuff of dome of bladder removed extraperitonealy preserving the umbilicus Histopahological report of excised tract also confirmed for urachal tissue. Post operative period on 12 month follow up patient is doing fine with no abdominal wall abnormality.

\section{Case Report-3}

A 30 year male presented to our OPD with history of recurrent purulent, cheesy discharge from umbilicus since adolescence. At the time of presentation patient was afebrile. The physical examination revealed periumbilical tenderness, erythema and discharge from the umbilicus. There were no signs of general infection. The laboratory tests were all within the normal limits.Urine culture was sterile. On ultra sound hypoechoeic area of size $3 \times 3 \mathrm{~cm}$ with echogenic particles inside present. On further investigation with CECT abdomen revealed sinus tract of size $1.2 \mathrm{~cm}$ with external opening at the umbilicus was present. Preoperative cystoscopy shows normal bladder finding. Provisional diagnosis was infected urachal sinus. Excision of the tract with median ligament and cuff of bladder was removed. Follow up at 12 month patient is doing fine with no fresh complaints.

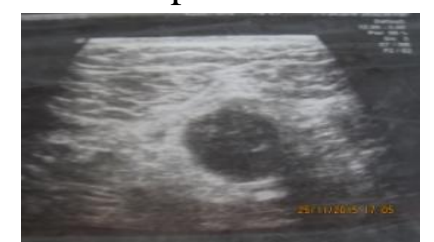

Fig -1 Ultra sonography showing hypoechoic mass with echogenic particles

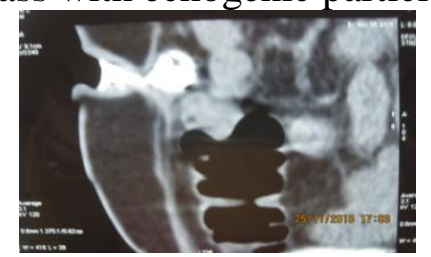

Fig-2 CT Sinogram showing Urachalsinus.

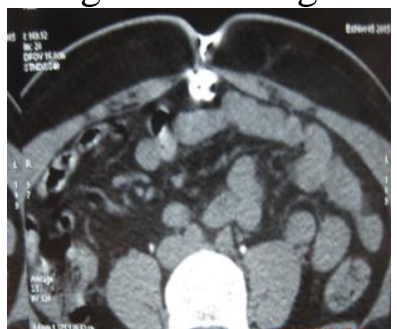

Fig -3 CT film showing sinus tract.

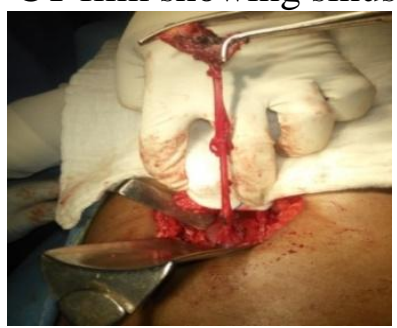

Fig -4 Removal of sinus tact with median ligament with bladder cuff. 


\section{Discussion}

The allantois develops as an extraembryonic cavity from the yolk sac and connects with the cranioventral portion of the cloaca, the future bladder. Around the 4th to 5th month of gestation, the allantoic duct and ventral cloaca involute as the bladder descends into the pelvis. The descent causes the allantoic duct to elongate because it does not grow with the embryo. This epithelialized fibromuscular tube continues to become narrower until it obliterates into a thick fibrous cord, the urachus (Moore, 1982) ${ }^{[1]}$. A partial or total defect of obliteration of the urachus channel after the fifth month of gestation can be the origin of urachal abnormalities.

Congenital anomalies of the urachus are

- Urachal sinus -15\%

- Urachal cyst $-30 \%$

- Patent urachus-50\%

- Vesicourachal diverticulum-5\%

This entity is usually discovered in childhood, but a late onset in adulthood is always possible. at Few cases of infected urachal remnants in adulthood were reported in the literature ${ }^{[2],[3]}$. In the umbilical-urachus sinus, the urachus obliterates at the bladder level but remains open at the umbilical site, causing a continuously draining sinus. In adults with persistant urachus $66 \%$ had hematuria or pain and $90 \%$ underwent excision. Surgical treatment in children consisted of simple excision, whereas over $50 \%$ of adults required partial or radical cystectomy because of malignancy $^{[4]}$. A recent report showed that children are more likely to have an infected urachal cyst, while adults are more likely to have an infected urachal sinus ${ }^{[5]}$.

Urachal remnants are clinically silent because the urachus is surrounded by the umbilicovesical fascia, so disease processes usually remain contained inside the pyramid-shaped space ${ }^{[6]}$. In the umbilical-urachus sinus, the urachus obliterates at the bladder level but remains open at the umbilical site, causing a continuously draining sinus. The manifestation is similar to that of the patent urachus. The presence of a persistent omphalomesenteric duct has to be considered. This would manifest as a Meckel diverticulum connected to the umbilicus. These structures can be very difficult to differentiate from an umbilical-urachus sinus because no connection to the bladder or bowel can be seen on sinugram.

Signs of inflammation becomes presenting feature when it is associated with infection. In our series one case was presented with acute infection to emergency while other two are presented to outpatient clinic. All patients are evaluated initially by ultrasound than by CECT abdomen. Cystoscopy done in all patients to rule out any bladder anamoly. All patients subjected to excision of tract with median umbilical ligament with removal of dome of bladder with preservation of umbelicus as in adult urachal remnants are associated with carcinoma ${ }^{[8],[9]}$.

In this series a transverse or midline infraumbilical incision was given in all cases. Mean operative time is 45 minutes, mean post hospital stay is 6 days. Patients were followed for 24 months. Laparoscopic approach for resection of the urachus was first introduced by Trondsen et al ${ }^{[10]}$. Since then some other teams have published their series of a laparoscopic approach ${ }^{[11],[12]}$.

\section{Conclusion}

Umbelical urachal sinus is a variant of urachal remnant presentation. Presentation in adults is a rare phenomenon but this have to kept in mind while evaluating acute abdomen. History taking and detailed physical examination may help in early diagnosis. CT scan is the gold standard diagnostic tools for suspected cases of urachal lesions. In adults complete tract excision with bladder cuff removal is the treatment of choice.

\section{References}

1. Moore KL. The urogenital system. Moore KL. The developing human. 3rd ed. Saunders: Philadelphia; 1982:255-297.

2. Ash A, Gujral R, Raio C. Infected urachal cyst initially misdiagnosed as an 
incarcerated umbilical hernia. J Emerg Med. 2012;42:171-173. doi: 10.1016/j.jemermed.2011.05.046.

3. Walker C. A case report of urachal abscess: a rare differential in adult abdominal pain. Hawaii Med J. 2010;69:35-36.

4. Ashley RA, Inman BA, Routh JC, et al. Urachal anomalies: a longitudinal study of urachal remnants in children and adults. $J$ Urol. 2007;178:1615-1618.

5. Iuchtman M, Rahav S, Zer M, Mogilner J, Siplovich L. Management of urachal anomalies in children and adults. Urology. 1993;42:426-430. doi: 10.1016/00904295( 93) $90375 \mathrm{~K}$.

6. Hammond G, Yglesis L, David JE. The uracus, its anatomy and associated fascia. Anat Rec.1941;80:271-274.

7. Galati V, Donovan B, Ramji F, Campbell J, Kropp BP, Frimberger D. Management of urachal remnants in early childhood. J Urol.2008;180:18241827.doi:10.1016/j.jur o.2008.03.105.

8. Goldman L, Caldamone A, Gauderer M, Hampel N, Wesselhoeft CW, Elder JS. Infected urachal cysts: a review of 10 cases. J Urol. 1988;140:375.

9. Mesrobian HG, Zacharias A, Balcom AH, Cohen RD. Ten years of experience with isolated urachal anomalies in children. $\mathrm{J}$ Urol. 1997;158:1316-1318. doi: 10.1016/S00225347(01)644653

10. Yohannes P, Bruno T, Pathan M, Baltaro R. Laparoscopic radical excision of urachal sinus. J Endourol. 2003;7:475-479

11. Stone NN, Garden RJ, Weber H. Laparoscopic excision of a urachal cyst. Urology. 1995;45:161-164. doi: 10.1016/S00904295(95)978240.

12. Okegawa T, Odagane A, Nutahara K, Higashihara E. Laparoscopic management of urachal remnants in adulthood. Int $\mathbf{J}$ Urol. 2006;13:1466-1469. doi: $10.1111 / \mathrm{j} .14422042 .2006 .01613$. 